\title{
Erratum to: Eukaryotic diversity in premise drinking water using 18S rDNA sequencing: implications for health risks
}

\author{
Helen Y. Buse • Jingrang Lu • Ian T. Struewing • \\ Nicholas J. Ashbolt
}

Published online: 2 March 2014

(C) Springer-Verlag Berlin Heidelberg 2014

\section{Erratum to: Environ Sci Pollut Res (2013) 20:6351-6366 DOI 10.1007/s11356-013-1646-5}

Because some of the eukaryotic species listed in Table 1 under "Algae" and "Dinoflagellates" were incorrectly grouped into those categories, we have listed them below under the correct grouping.

Erratum (I): Table 1 Sequence homologies and number of clones obtained for each major representative eukaryotic group
Erratum (II): Fig 2. Relative abundance of $18 \mathrm{~S}$ rDNA clone in various eukaryotic groups.

The above listed species were of low abundance in our clone libraries therefore discussion of our results was not affected by these changes as well as the major findings of our study.

The authors would like to thank Dr. René Groben, Matis ohf., Food and Biotech R\&D, for bringing the error to our attention.

Table 1 Sequence homologies and number of clones obtained for each major representative eukaryotic group

\begin{tabular}{|c|c|c|c|c|c|}
\hline Group & Sequence length (bp) & Accession no. ${ }^{\mathrm{a}}$ & Closest Relative & no. clones & $\%$ Similarity $^{\mathrm{b}}$ \\
\hline \multicolumn{6}{|l|}{ Algae } \\
\hline & 613 & HQ912625 & Cyclotella sp. L04-2 & 16 & $99 \%$ \\
\hline \multicolumn{6}{|c|}{ Dinoflagellates } \\
\hline & 629 & JN615412 & Amphidoma languida strain SMI & 1 & $91 \%$ \\
\hline & 615 & HQ324899 & Azadinium poporum isolate UTHD4 & 2 & $91 \%$ \\
\hline & 677 & EF492492 & Gymnodinium sp. CCMP425 & 92 & $98 \%$ \\
\hline & 614 & AJ415515 & Scrippsiella trochoidea & 1 & $93 \%$ \\
\hline & 614 & AY443025 & Woloszynskia leopoliensis & 3 & $98 \%$ \\
\hline & 701 & EF058253 & Woloszynskia pascheri strain CCAC0075 & 37 & $99 \%$ \\
\hline \multicolumn{6}{|l|}{ Misc } \\
\hline & 617 & EF455761 & Malawimonas jakobiformis & 9 & $99 \%$ \\
\hline & 601 & FJ424512 & Parvilucifera prorocentri & 1 & $78 \%$ \\
\hline
\end{tabular}

a, Accession no. for closest relative

b, For each clone sequence, query coverage range, $74-100 \%$, and e value range, $0.0-1 \mathrm{e}^{-17}$

The online version of the original article can be found at http://dx.doi.org/

10.1007/s11356-013-1646-5.

H. Y. Buse $(\bowtie) \cdot$ I. T. Struewing

Dynamac c/o US Environmental Protection Agency, 26 W Martin

Luther King Drive, Cincinnati, OH 45242, USA

e-mail: buse.helen@epa.gov

J. Lu $\cdot$ N. J. Ashbolt

National Exposure Research Laboratory, Office of Research and

Development, US Environmental Protection Agency, Cincinnati,

$\mathrm{OH}$, USA 


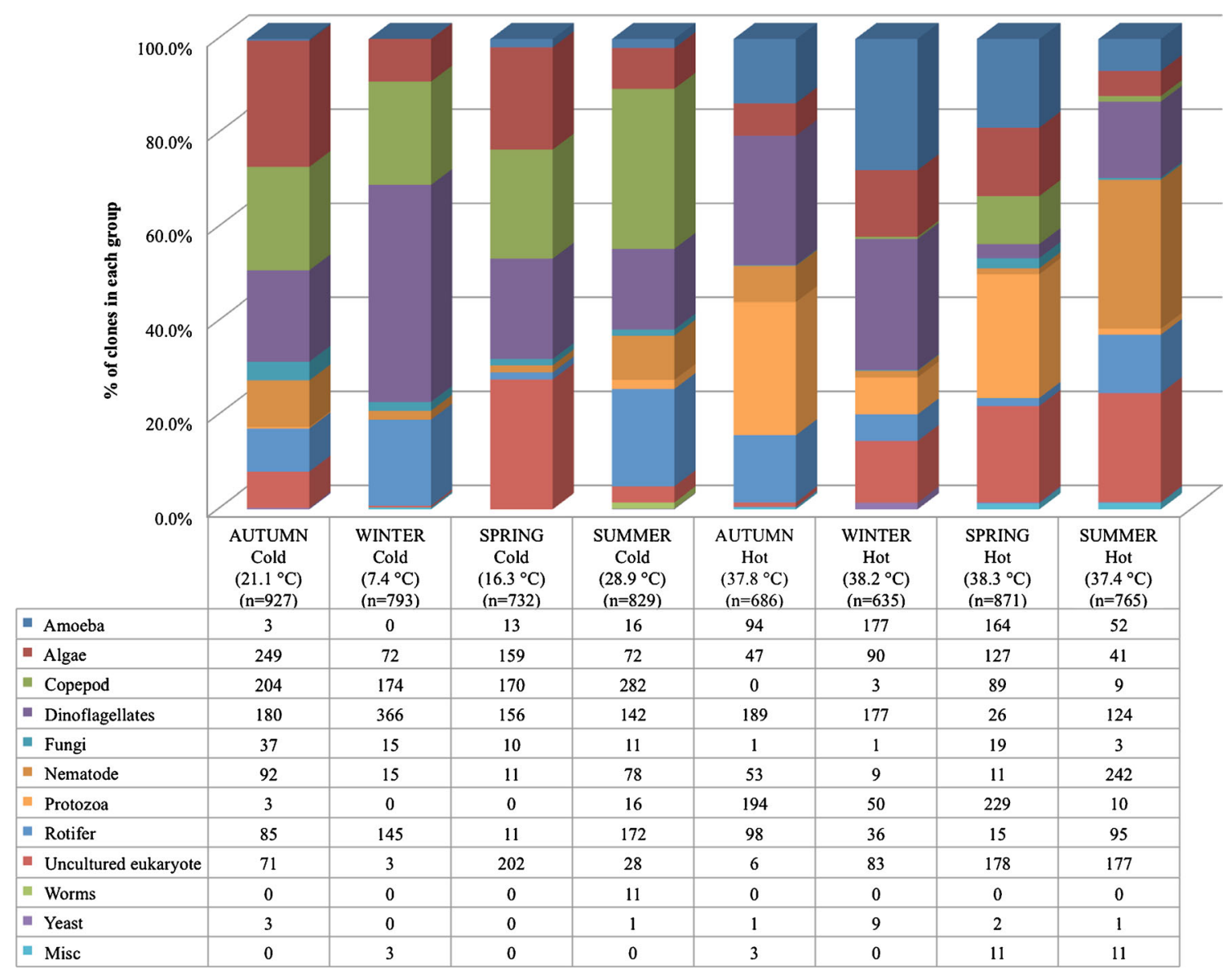

Fig. 2 